Symmetries, Differential Equations and Applications (SDEA-II)

International Journal of Modern Physics: Conference Series

Vol. 38 (2015) 1502004 (1 page)

DOI: $10.1142 / \mathrm{S} 2010194515020048$

\title{
Peer Review Statement
}

\begin{abstract}
All articles in this volume have been subjected to peer review administered by the proceedings editors and have been revised in accordance with comments of the expert referees.
\end{abstract}

